\title{
A unified view on heterogeneous and homogeneous catalysts through a combination of spectroscopy and quantum chemistry $\dagger$
}

\author{
Dimitrios Maganas, ${ }^{a}$ Annette Trunschke, ${ }^{b}$ Robert Schlögl ${ }^{* a b}$ \\ and Frank Neese ${ }^{\star a}$
}

Received 25th November 2015, Accepted 4th January 2016

DOI: $10.1039 / \mathrm{c} 5 \mathrm{fd0} 0193 \mathrm{e}$

Identifying catalytically active structures or intermediates in homogeneous and heterogeneous catalysis is a formidable challenge. However, obtaining experimentally verified insight into the active species in heterogeneous catalysis is a tremendously challenging problem. Many highly advanced spectroscopic and microscopic methods have been developed to probe surfaces. In this discussion we employ a combination of spectroscopic methods to study two closely related systems from the heterogeneous (the silica-supported vanadium oxide $\mathrm{VO}_{x} / \mathrm{SBA}-15$ ) and homogeneous (the complex $\mathrm{K}\left[\mathrm{VO}\left(\mathrm{O}_{2}\right) \mathrm{Hheida}\right]$ ) domains. Spectroscopic measurements were conducted strictly in parallel for both systems and consisted of oxygen $\mathrm{K}$-edge and vanadium L-edge $\mathrm{X}$-ray absorption measurements in conjunction with resonance Raman spectroscopy. It is shown that the full information content of the spectra can be developed through advanced quantum chemical calculations that directly address the sought after structure-spectra relationships. To this end we employ the recently developed restricted open shell configuration interaction theory together with the time-dependent theory of electronic spectroscopy to calculate XAS and rR spectra respectively. The results of the study demonstrate that: (a) a combination of several spectroscopic techniques is of paramount importance in identifying signature structural motifs and (b) quantum chemistry is an extremely powerful guide in cross connecting theory and experiment as well as the homogeneous and heterogeneous catalysis fields. It is emphasized that the calculation of spectroscopic observables provides an excellent way for the critical experimental validation of theoretical results.

\footnotetext{
${ }^{a}$ Max Planck Institute for Chemical Energy Conversion, Stiftstr. 34-36, D-45470 Mülheim an der Ruhr, Germany.E-mail: Frank.Neese@cec.mpg.de; rs01@fhi-berlin.mpg.de

${ }^{b}$ Fritz-Haber Institut, Faradayweg 4-6, 14195 Berlin, Germany

$\dagger$ Electronic supplementary information (ESI) available: Experimental and fitting procedure, computational details, electronic structure, Fig. S1-S9, Table S1, and bibliography. See DOI: 10.1039/c5fd00193e
} 


\section{Introduction}

The impact of heterogeneous catalysis on industrial scale processes hardly needs to be emphasized. In fact the majority of catalytic processes that are implemented on a large-scale industrial basis, are based on heterogeneous catalysis. ${ }^{1}$ Developing new catalysts that are efficient, stable and selective is a major challenge. Rather than finding such systems through trial and error, one would hope to take a rational approach that is based on a detailed understanding of the catalytic mechanism. To achieve the latter requires obtaining insight into the reactive species that exist on surfaces under in operando conditions. ${ }^{2}$

Theoretical calculations are in very widespread use in order to obtain insight into heterogeneous catalysts. The theoretical treatment of surfaces and solids is strongly dominated by the method of density functional theory (DFT). ${ }^{3}$ While this method has definite and important strengths and advantages, it also suffers from some significant shortcomings, as will be elaborated below. In many cases the connection between theory and experiment is rather indirect. For example, volcano plots are frequently used to correlate structure and reactivity by connecting substrate binding energies at ideal surfaces with kinetic rates. ${ }^{4,5}$ Due to several strong assumptions needed for the connection between thermodynamics, kinetics and mechanism, the necessity for a more immediate connection between theory and experiment emerges. It is the aim of this paper to emphasize that such strong ties between theory and experiment can be established by combining a range of spectroscopic methods with the results of carefully calibrated theoretical spectroscopy.

Combined experimental and theoretical multi-method protocols have been used to great advantage in (bio)inorganic chemistry and catalysis. For example, a theoretical spectroscopy approach has proven instrumental in characterizing exotic reactive species in model systems (e.g. genuine $\mathrm{Fe}(\mathrm{v})^{6}$ and the first $\left.\mathrm{Fe}(\mathrm{vI}) \operatorname{complex}^{7}\right)$, enzymes $^{8-10}($ e.g. the identification of a unique carbide center in the active site of the dinitrogen activating enzyme nitrogenase ${ }^{11}$ ), or in clarifying the structure ${ }^{12}$ and oxidation states ${ }^{13}$ of the oxygen evolving complex in photosynthesis. It should be emphasized that in order to successfully apply a theoretical spectroscopy approach, it is of utmost importance that the theoretical methods are properly calibrated, that is, the error bars of the theoretical predictions must be known from studying a series of known and understood systems. Hence, all predictions of theoretical methods are only valid within the confidence intervals defined by the calibration procedure. All contemporary calculations of homogeneous and heterogeneous systems likewise are afflicted with methodological errors and errors stemming from the inevitable oversimplification of real systems. It is for this reason that one is well advised to connect theory with as many spectroscopic experiments as one can possibly obtain. It should be emphasized that, once broad agreement between theory and experiment has been achieved, one can: (a) cross correlate structurespectra relationships between vastly different experimental methods and (b) obtain experimentally calibrated insight into not only the geometric but also the allimportant electronic structure of the systems under investigation. The latter is instrumental for obtaining deep insight into the reactivity of the system. This might be impressively exemplified with the case of the reactive $\mathrm{Fe}(\mathrm{Iv})-\mathrm{oxo}(\mathrm{FeO})^{2+}$ core, the complex reactivity of which might well be considered as understood., ${ }^{\mathbf{9}, 10}$ 
In this work, we will try to establish a strong connection between the domains of homogeneous and heterogeneous catalysis by using parallel spectroscopic protocols for a heterogeneous system, namely silica supported vanadia catalysts, ${ }^{14,15}$ and a closely related homogeneous system, the coordination complex $\mathrm{K}\left[\mathrm{VO}\left(\mathrm{O}_{2}\right)\right.$ Hheida]. The chemical importance of these systems is presented in the $\mathrm{ESI} \dagger$ while representative structures are shown in Fig. 1.

The experimental methods that were chosen for the current study involve vanadium L-edge and oxygen K-edge X-ray absorption (XAS) spectroscopy, absorption (ABS) and resonance Raman (rR) spectroscopy. The theoretical methodology required to calculate L-edge XAS and rR spectra is not part of the standard arsenal of theoretical solid state physics or quantum chemistry. In fact, both techniques require extensive method development. In particular, the field of L-edge spectroscopy cannot be successfully approached on the basis of DFT at all. Hence, our theoretical approaches deviate strongly from the currently employed DFT methods in that they are largely based on ideas from wavefunction-based $a b$ initio quantum chemistry. We emphasize the properties of the $\mathrm{N}$-particle states of the system ( $N$ being the number of electrons) in favour of the ubiquitous oneparticle (orbital) picture that is implied by interpretation based on DFT. The drawback of focusing on wavefunction approaches is that truly periodic calculations are not available for the approaches that we are using. The next best alternative, that we follow, is to employ embedded clusters. Once the clusters are sufficiently large, the results of the periodic calculations are approached. However, convergence with cluster size is an issue that must be carefully monitored. Fortunately, cluster calculations with a few hundred atoms are feasible with modern hard- and software technologies such that the limitations of using cluster models are not nearly as severe as they were in the past.

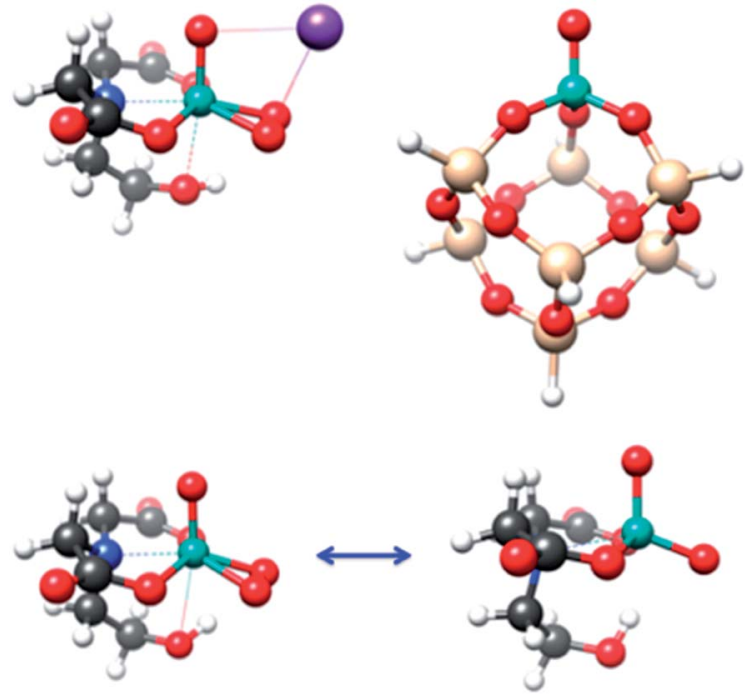

Fig. 1 Top left: The molecular structure of $\mathrm{K}\left[\mathrm{VO}\left(\mathrm{O}_{2}\right) \mathrm{Hheida]}\right.$. Top right: Representative model structure of monomeric silica supported vanadia catalyst, $\mathrm{VSi}_{7} \mathrm{H}_{7} \mathrm{O}_{13}$. Bottom: Two possible active forms of the molecular catalyst; $\left[\mathrm{VO}\left(\mathrm{O}_{2}\right) \mathrm{Hheida}\right]^{-}$(left) and oxygen deficient [VO(O)Hheida] $]^{-}$(right). Atom colouring: $\mathrm{H}$ (white), C (gray), O (red), N (blue), V (cyan), Si (light yellow) and K (purple). 
We have recently introduced the restricted open shell configuration interaction singles (ROCIS) method as a method of general applicability to interpret metal L-edge spectra of a large class of molecules, ranging from mononuclear complexes up to polymetallic clusters. ${ }^{16-19}$ The method is able to predict metal L-edge spectra for a variety of metal complexes in different oxidation states and coordination environments. In addition it has been calibrated for a large set of vanadium mononuclear complexes and has proven successful in interpreting $\mathrm{V}$ L-edge and $\mathrm{O}$ K-edge spectra of $\mathrm{V}_{2} \mathrm{O}_{5}$ (ref. 16) as well as the Ti L-edge spectra of $\mathrm{TiO}_{2}$ (rutile and anatase) and Ca L-edge spectra of $\mathrm{CaF}_{2}$ solids. ${ }^{20}$ Furthermore, we have also presented a highly efficient method to treat the band-shape of optical absorption spectra and resonance Raman spectra for large molecules..$^{\mathbf{2 1 2 2}}$

\section{Experimental section}

\section{Synthesis}

$\mathrm{VO}_{x} / \mathrm{SBA}-15$ catalysts (V loading of $2-14 \% \mathrm{wt} \% / 0.6-13.7 \mathrm{~V}$-atoms $\mathrm{nm}^{-2}$ ) were prepared by an ion-exchange approach as described elsewhere. ${ }^{23}$ Further details
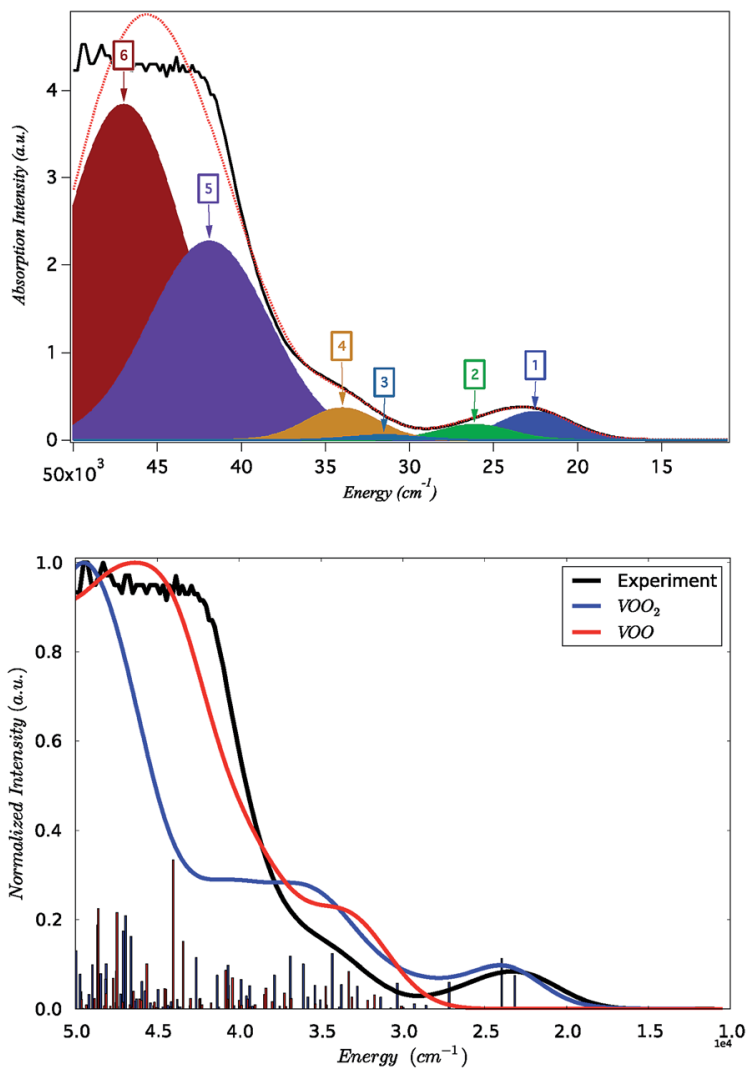

Fig. 2 Black line: experimental absorption spectrum of $\mathrm{K}\left[\mathrm{VO}\left(\mathrm{O}_{2}\right) \mathrm{Hheida]}\right.$. Top: Gaussian deconvolution of the absorption spectrum. The Gaussian fit is constrained to constant widths FWHM $=4000$ and $7500 \mathrm{~cm}^{-1}$ for bands $1-4$ and 5-6 respectively. Bottom: B3LYP TD-DFT calculated spectra for $\left[\mathrm{VO}\left(\mathrm{O}_{2}\right) \mathrm{Hh}_{\text {heidal }}\right]^{-}$(blue) and $[\mathrm{VO}(\mathrm{O}) \mathrm{Hheida}]^{-}$(red) complexes. Blue and red sticks indicate the corresponding calculated non-relativistic states. 
regarding spectroscopic characterization and theoretical calculations are summarized in the ESI. $\dagger$

\section{Spectroscopic data}

The absorption spectrum of $\mathrm{K}\left[\mathrm{VO}\left(\mathrm{O}_{2}\right)\right.$ Hheida] is presented in Fig. 2. It is characterized by a low energy and a broad signal located at $26000 \mathrm{~cm}^{-1}$, as well as by a higher energy shoulder showing a maximum at $34000 \mathrm{~cm}^{-1}$. This later signal grows at the rising edge of an even broader and partially saturated signal centered at $45000 \mathrm{~cm}^{-1}$. On the other hand the absorption spectrum of $\mathrm{VO}_{x} / \mathrm{SBA}-15$ (vanadium loading $2 \%$ and $8 \%$ ) is presented in Fig. S2. $\dagger$ It is characterized by a low energy shoulder located at $25000 \mathrm{~cm}^{-1}$ followed by a high-energy broad signal centered at about $40000 \mathrm{~cm}^{-1}$. It should be noted that the absorption spectra show pronounced changes at low vanadium loadings, in fact the spectrum manifold shifts to higher energies as the vanadium loading decreases. This phenomenon merits further investigation.

Furthermore, for both catalytic systems Raman spectra were obtained under near resonance conditions. For the molecular complex, this was achieved using a $532 \mathrm{~nm}$ laser. ${ }^{24}$ As can be seen in Fig. 3 the spectrum shows characteristic enhanced $\mathrm{rR}$ bands located at 970, 922, 569, 556, 509 and $485 \mathrm{~cm}^{-1}$. The corresponding Raman spectrum of dehydrated $\mathrm{VO}_{x} / \mathrm{SBA}-15$ at low vanadium loading (about $2 \%$ ), was obtained upon excitation ${ }^{25,26}$ with a $488 \mathrm{~nm}$ laser $^{23}$ and is presented in Fig. S2. $\dagger$ For such a low vanadium loading value, the only Raman active signal with substantial intensity is located in the region between $950-1100 \mathrm{~cm}^{-1}$. This signal has been assigned to a variety of $\mathrm{V}, \mathrm{O}$ and Si functionalities ranging between monomeric to oligomeric vanadia species as are shown in Scheme 1..$^{14,25,27-33}$ However, no consensus has emerged. It is clear that based on these data, this signal can only be assigned to a mixture of overlapping features of monomeric and oligomeric vanadyl species coexisting on SBA-15 already at small vanadium loadings (e.g. $0.6 \mathrm{~V}$ atoms per $\mathrm{nm}^{2}$ ).

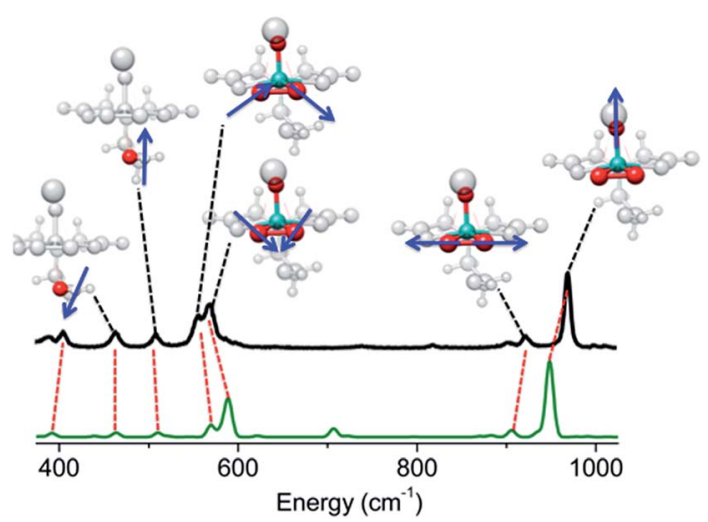

Fig. 3 Experimental (black) rRaman spectrum of $\mathrm{K}\left[\mathrm{VO}\left(\mathrm{O}_{2}\right)\right.$ Hheida] observed at 18700 $\mathrm{cm}^{-1}$ excitation energy $(532 \mathrm{~nm}$ ). Calculated (green) TD-DFT rRaman spectrum. Coloured groups and blue arrows indicate the active vibrational modes dominating certain experimental (and calculated) signals. 


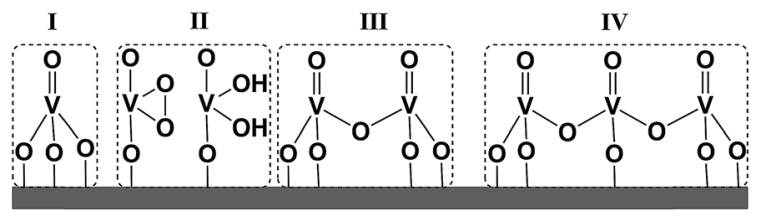

SBA-15

Scheme 1 Four possible scenarios for $\mathrm{VO}_{x}$ growing over a 2D silica surface (SBA-15). (I) Vanadyl type, (II) umbrella type, (III) dimeric and (IV) oligomeric species.

In addition, the experimental V L-edge and O K-edge XAS spectra for $\mathrm{K}\left[\mathrm{VO}\left(\mathrm{O}_{2}\right)\right.$ Hheida] and $\mathrm{VO}_{x} / \mathrm{SBA}-15$ at various vanadium loadings are presented in Fig. 4 and 5 , respectively. In general $\mathrm{VO}_{x}$ species are characterized by characteristic $\mathrm{V}$ L-edge and $\mathrm{O}$ K-edge spectra. ${ }^{34,35}$ In particular, for the case of the $\mathrm{K}\left[\mathrm{VO}\left(\mathrm{O}_{2}\right)\right.$ Hheida $]$ complex the $\mathrm{V} \mathrm{L}_{3}$-edge spectrum is characterized by an intense feature located at $518 \mathrm{eV}$, as well as three shoulders located at 515, 516 and $519 \mathrm{eV}$. The $\mathrm{V} \mathrm{L}_{2}$-edge spectrum is even broader showing two features centered at 523 and $524 \mathrm{eV}$. It

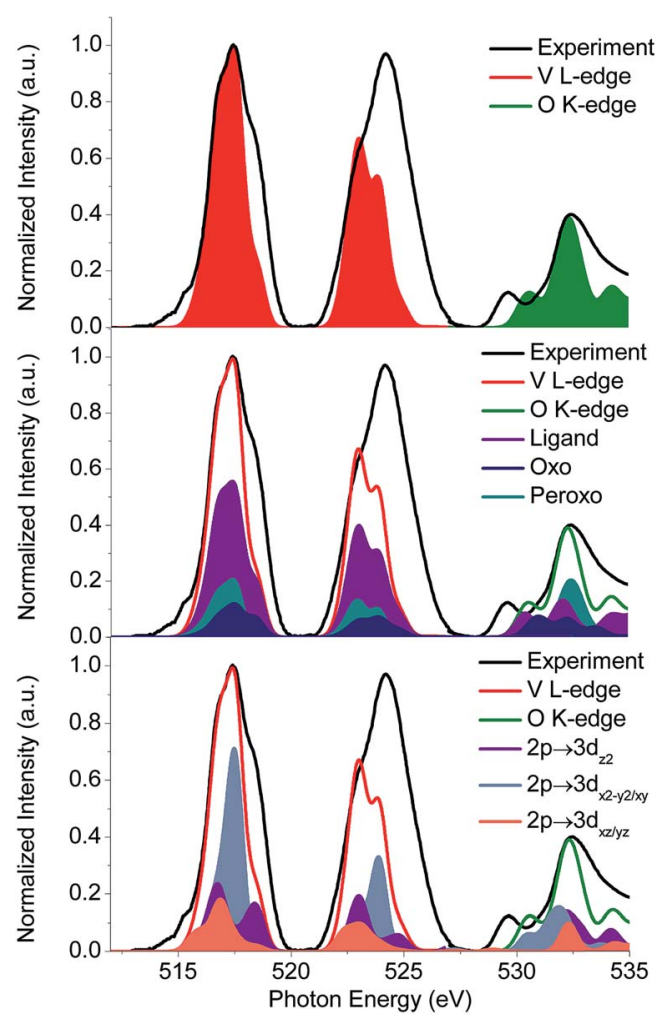

Fig. 4 Black line: experimental V L-edge and O K-edge X-ray absorption spectra. Red and green lines and areas correspond to the B3LYP/ROCIS calculated spectra for $80 \%\left[\mathrm{VO}\left(\mathrm{O}_{2}\right)\right.$ Hheida] $+20 \%$ [VO(O)Hheida] active species composition (top). Deconvolution of the calculated spectra in terms of participating functional groups (middle) and single electron excitations (bottom). 


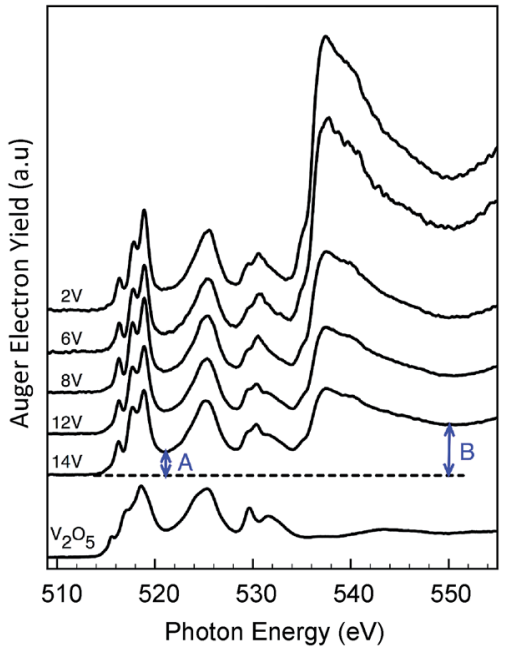

Fig. $5 V L_{3,2}$ and $O K-N E X A F S$ of dehydrated $V_{x} / S B A-15$ catalysts with various $V$ loadings as indicated. Spectra were recorded in $50 \mathrm{PaO}_{2}$ at $673 \mathrm{~K}$. A reference spectrum of $\mathrm{V}_{2} \mathrm{O}_{5}$ powder is shown as well. The $V$ edge jump " $A$ " and the total edge jump " $B$ " are marked with arrows.

must be emphasized that in total the $\mathrm{V}$ L-edge spectrum reveals a much broader bandwidth with respect to the corresponding spectra of other oxo-peroxo $\mathrm{V}^{(\mathrm{V})}$ complexes $^{17}$ indicating the coexistence of more than one species, as will be
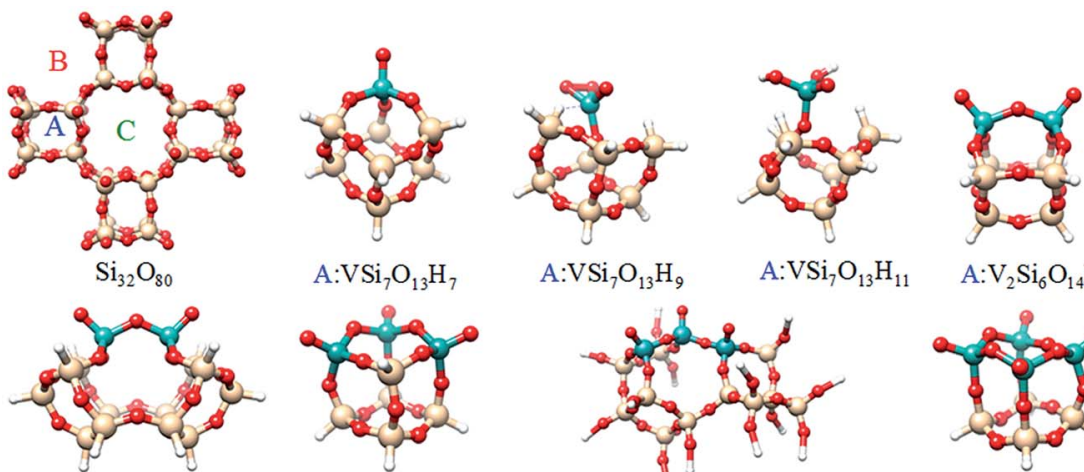

$\mathrm{A}: \mathrm{VSi}_{7} \mathrm{O}_{13} \mathrm{H}_{7}$

$\mathrm{A}: \mathrm{VSi}_{7} \mathrm{O}_{13} \mathrm{H}_{9}$

$\mathrm{A}: \mathrm{VSi}_{7} \mathrm{O}_{13} \mathrm{H}_{11}$

$\mathrm{A}: \mathrm{V}_{2} \mathrm{Si}_{6} \mathrm{O}_{14} \mathrm{H}_{6}$
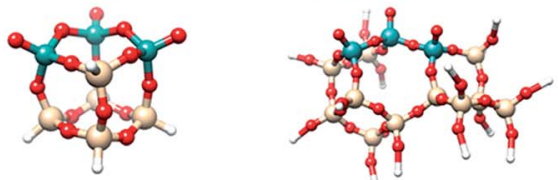

$\mathrm{C}: \mathrm{V}_{3} \mathrm{Si}_{11} \mathrm{O}_{37} \mathrm{H}_{15}$

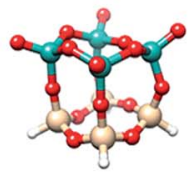

$\mathrm{B}: \mathrm{V}_{2} \mathrm{Si}_{12} \mathrm{O}_{23} \mathrm{H}_{12} \quad \mathrm{~A}: \mathrm{V}_{3} \mathrm{Si}_{5} \mathrm{O}_{15} \mathrm{H}_{5}$
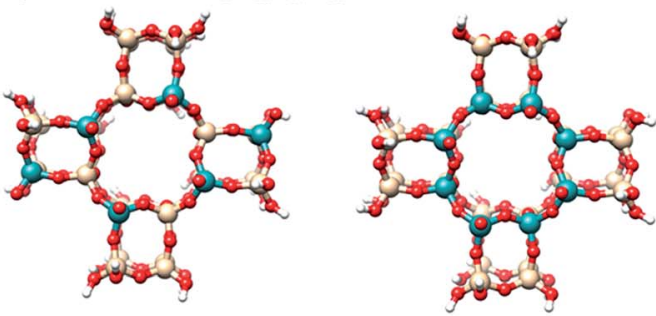

$\mathrm{C}: \mathrm{V}_{4} \mathrm{Si}_{28} \mathrm{O}_{80} \mathrm{H}_{28}$

$\mathrm{A}, \mathrm{C}: \mathrm{V}_{6} \mathrm{Si}_{26} \mathrm{O}_{80} \mathrm{H}_{28}$

$\mathrm{C}: \mathrm{V}_{8} \mathrm{Si}_{24} \mathrm{O}_{80} \mathrm{H}_{28}$

Fig. 6 Graphical representation of the cluster models used to model the different silica supported vanadia centers. More information is provided in the ESI (Fig. S1 $\uparrow$ ). 
analyzed in the theoretical section. Similarly, the O K-edge spectrum also has two broad features located at 529 and $534 \mathrm{eV}$.

The corresponding spectra of VOx/SBA-15 are presented in Fig. 5 . In the $\mathrm{V} \mathrm{L}_{3}$ region three distinguishable signals are observed at 516, 517.5 and $519 \mathrm{eV}$ while in the $\mathrm{V} \mathrm{L}_{2}$ region only one broad signal is observed at $525 \mathrm{eV}$. In addition the $\mathrm{O}$ K-edge spectrum contains a dominant signal around $530 \mathrm{eV}$ with two broad shoulders located at 529.5 and $531 \mathrm{eV}$. These spectra differ substantially from the respective $\mathrm{V}$ L-edge and $\mathrm{O}$ K-edge spectra of crystalline $\mathrm{V}_{2} \mathrm{O}_{5}$ shown also in Fig. 5 (bottom). In fact, the $\mathrm{V}$ L-edge spectrum of $\mathrm{V}_{2} \mathrm{O}_{5}$ is broader due to bulk effects originating from weak $\mathrm{O}-\mathrm{V} \cdots \mathrm{O}-\mathrm{V}$ interactions within the bilayer crystallographic structure. ${ }^{16}$ Previously, theoretical investigations have concluded that a mixture of monomeric and oligomeric vanadyl species dominates the $\mathrm{O}$ K-edge spectrum. ${ }^{36}$ On the other hand, recent studies on ceria supported vanadia species have suggested that at low vanadium loadings $\left(0.7 \mathrm{~V}\right.$ atoms per $\left.\mathrm{nm}^{2}\right)$ there is a uniform coverage of the catalyst with trimeric $\mathrm{V}$ species. ${ }^{37}$

In the next section a systematic computational study of all these properties will be carried out based on model structures described in the ESI and shown in Fig. 6 and $\mathrm{S} 1 . \dagger$

\section{Theoretical calculations}

\section{Absorption spectroscopy}

On the basis of the particle/hole excitation patterns shown in Fig. 7 and $\mathrm{S} 3 \uparrow$ the absorption spectrum of $\left[\mathrm{VO}\left(\mathrm{O}_{2}\right) \text { Hheida }\right]^{-}$can be satisfactorily fitted with a minimum of six Gaussian bands. In the second step of the analysis we perform TD-DFT calculations of the absorption spectra assuming the coexistence of $\left[\mathrm{VO}\left(\mathrm{O}_{2}\right) \text { Hheida }\right]^{-}$and the oxygen deficient $[\mathrm{VO}(\mathrm{O}) \text { Hheida }]^{-}$complexes. The results are shown in Fig. 2 (bottom). Evidently, the signal located at $26000 \mathrm{~cm}^{-1}$ is

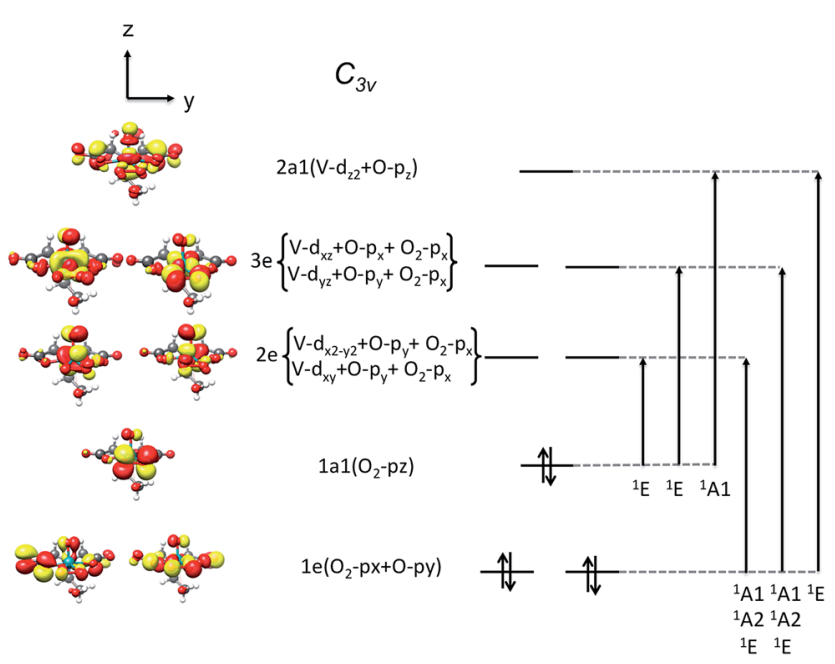

Fig. 7 The $\mathrm{MO}$ splitting diagram of the local $\mathrm{V}-\mathrm{OO}_{2}\left(C_{3 \mathrm{v}}\right)$ core. In addition, the adapted $2 p-3 d$ transitions in the one electron particle/hole approximation are visualized assuming ideal $C_{3 v}$ ligand field splitting. 
solely dominated by the $\mathrm{V}-\mathrm{OO}_{2}$ functional group. In fact the Gaussian bands 1 and 2 correspond to the $\mathrm{LMCT}{ }^{1} \mathrm{E}\left(1 \mathrm{a}_{1} \rightarrow 2 \mathrm{e}\right),{ }^{1} \mathrm{E}\left(1 \mathrm{a}_{1} \rightarrow 3 \mathrm{e}\right)$ and ${ }^{1} A_{1}\left(1 \mathrm{a}_{1} \rightarrow 2 \mathrm{a}_{1}\right)$ one-electron excitations, respectively. On the other hand, the bands located at 34000 and $45000 \mathrm{~cm}^{-1}$ (Gaussians 3-6) are dominated by LMCT and Ligand to Ligand CT (LLCT) electron excitations arising from the $\mathrm{V}-\mathrm{OO}_{2}$ and $\mathrm{V}-\mathrm{OO}$ functional groups. Likewise the changes observed in the VOx/SBA-15 spectra (Fig. S2, $\dagger$ top-left) are nicely reproduced in the calculations, as shown in Fig. S2† (top-right). Indeed, by proceeding from monomeric to oligomeric species, there are spectral shifts at lower energies. As long as the vanadium loading remains limited (about $2 \%$ ), it is necessary to investigate whether a unique structure can primarily dominate the experimental spectra as will be shown below by analyzing the rRaman and XAS spectra.

\section{rRaman spectroscopy}

By setting the excitation frequency to pre-resonance with the absorption band at $26000 \mathrm{~cm}^{-1}$, e.g. an excitation frequency of $450 \mathrm{~nm}$, the rRaman bands of $\mathrm{K}\left[\mathrm{VO}\left(\mathrm{O}_{2}\right)\right.$ Hheida $]$ presented in Fig. 3 are calculated. We notice that certain deficiencies in the calculated energies of the band positions and relative intensities with respect to experiment are observed, most likely due to the fact that the IMDHO model does not account for terms of second or higher order between excited and ground vibrational Hamiltonians. ${ }^{21,22}$ Despite the fact that the agreement between theory and experiment is only a semiquantitative agreement for some of the rRaman bands, the IMDHO approach still enables a conclusive assignment of the experimental Raman bands. It should be noted that at this region of excitation energies, the observed absorption band is solely dominated by the $\left[\mathrm{VO}\left(\mathrm{O}_{2}\right) \text { Hheida }\right]^{-}$species. Following the discussion above, this band corresponds to LMCT states arising solely from the $\mathrm{V}-\mathrm{OO}_{2}$ functional group of the $\left[\mathrm{VO}\left(\mathrm{O}_{2}\right) \text { Hheida }\right]^{-}$complex. Due to the excellent agreement between theory and experiment the assignment of the rRaman spectral features is straightforward. The signals located at 970 and $922 \mathrm{~cm}^{-1}$ are assigned to the stretching vibrations of the $\mathrm{V}-\mathrm{O}$ (vanadyl-oxo) and the $\mathrm{O}-\mathrm{O}$ (peroxo) groups, respectively. In addition, the signals centered at 569 and $556 \mathrm{~cm}^{-1}$ are dominated by symmetric and antisymmetric $\mathrm{V}-\mathrm{O}$ (peroxo) stretching vibrations. Finally, the low intensity signals located at 509 and $565 \mathrm{~cm}^{-1}$ are dominated by hydroxyl stretching vibrations.

Similarly, the rRaman ${ }^{23,25,30}$ spectra were evaluated for low lying excited states of all the model structures representing VOx/SBA-15 in the range between 300$500 \mathrm{~nm}\left(20000-35000 \mathrm{~cm}^{-1}\right)$. The corresponding spectra are presented in Fig. S4 and $55 . \dagger$ This strategy was followed for all model structures and will be illustrated below for the $\mathrm{V}_{3} \mathrm{Si}_{5} \mathrm{H}_{5} \mathrm{O}_{15}$ structure. The calculated absorption spectrum is characterized by three low-lying groups of bands located at 22500 (orange), 25000 (cyan) and $27000 \mathrm{~cm}^{-1}$ (purple) depicted in Fig. 8 (bottom-left, higher lying states are drawn in gray). These bands originate from either single-electron excitations within the $\mathrm{V}=\mathrm{O}$ and $\mathrm{V}-\mathrm{O}-\mathrm{V}$ fragments (orange) and the $\mathrm{V}=\mathrm{O}$ and $\mathrm{Si}-\mathrm{O}-\mathrm{V}$ structural motifs (cyan and purple). Based on those calculated excitation energies the corresponding rRaman spectra can be calculated (Fig. 8, top-right).

The calculated rRaman spectra reproduce the observed signals around 1000 $\mathrm{cm}^{-1}$ and they are in very good agreement with previous studies. ${ }^{25,26,30}$ Inspection 


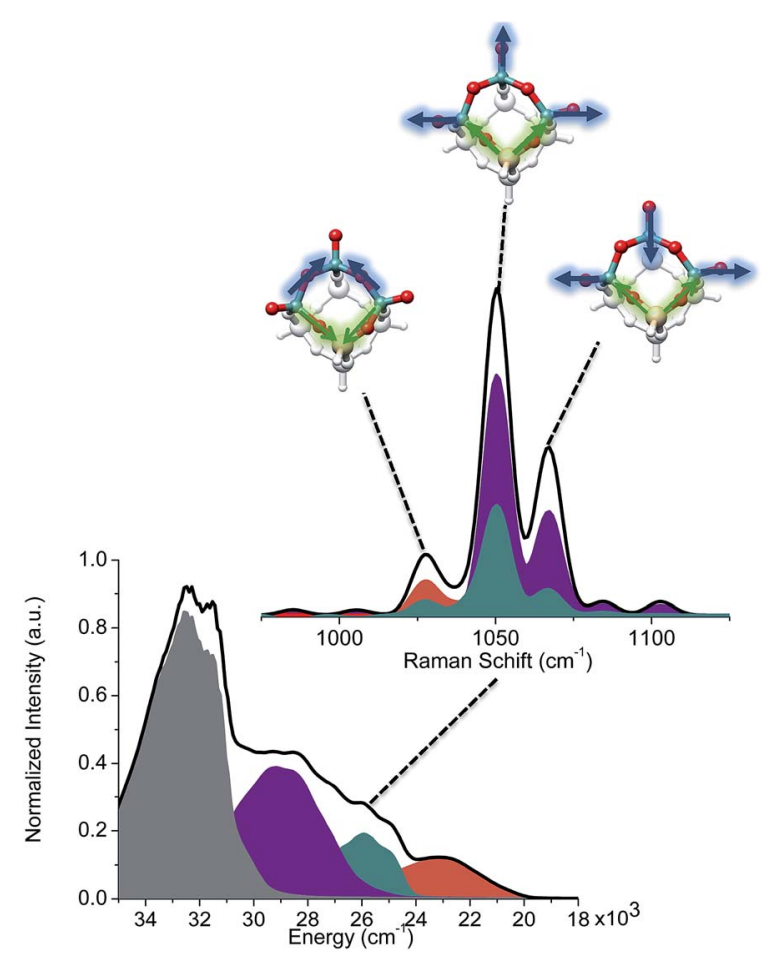

Fig. 8 Bottom-left: Calculated absorption spectra accounting for the band shape of the dominating states for $\mathrm{V}_{3} \mathrm{Si}_{5} \mathrm{H}_{5} \mathrm{O}_{15}$. The states are grouped according to the character of the dominating single-electron excitations involving either $\mathrm{V}=\mathrm{O}$ and $\mathrm{V}-\mathrm{O}-\mathrm{V}$ sequences (orange), or $\mathrm{V}=\mathrm{O}$ and $\mathrm{Si}-\mathrm{O}-\mathrm{V}$ sequences (cyan and purple). Top-right: Calculated Raman spectra. The spectra are calculated at an absorption energy of $26000 \mathrm{~cm}^{-1}$. Orange, cyan and purple bands indicate active vibrational modes pointed with arrows.

of Fig. S4 and S5 $\uparrow$ reveals that, although the presence of monomeric umbrella type structures cannot be excluded, they certainly do not dominate the intensity of the signal around $1000 \mathrm{~cm}^{-1}$, this conclusion follows from the absence of the characteristic $\mathrm{O}=\mathrm{O}$ and $\mathrm{Si}-\mathrm{O}-\mathrm{V}$ signals expected at $<1000 \mathrm{~cm}^{-1}$ that are key features for this type of structure. Based on these data it appears that the structures which contain vanadyl $\mathrm{V}=\mathrm{O}$ bonds and bridging $\mathrm{V}-\mathrm{O}-\mathrm{V}$ units are always in better agreement with experiment for the signals located around $1000 \mathrm{~cm}^{-1}$. At this stage of analysis, the Raman technique alone is not conclusive with respect to the dominant structural feature. Nevertheless, we note that the calculations for the trimeric structure presented in Fig. 8 provide a very good agreement with experiment. Analysis of the predominant vibrational modes indicates that the signal at around $1000 \mathrm{~cm}^{-1}$ is dominated by combinations of $\mathrm{V}-\mathrm{O}-\mathrm{V}$ and $\mathrm{V}-\mathrm{O}-\mathrm{Si}(1025$ $\mathrm{cm}^{-1}$ ) as well as $\mathrm{V}=\mathrm{O}$ and $\mathrm{V}-\mathrm{O}-\mathrm{Si}\left(1050,1075 \mathrm{~cm}^{-1}\right)$ stretching vibrations (Fig. 8, top-right). More information can be obtained by deconvoluting the calculated rRaman signals in terms of the dominating excited state. As can be seen in Fig. 8 (bottom-left) the group of states are heavily overlapping as each absorption band contains tails that overlap with the other two. The signal at $1025 \mathrm{~cm}^{-1}$ is mainly dominated by states involving $\mathrm{V}=\mathrm{O}$ and $\mathrm{V}-\mathrm{O}-\mathrm{V}$ (orange), as well as the $\mathrm{V}=\mathrm{O}$ and 


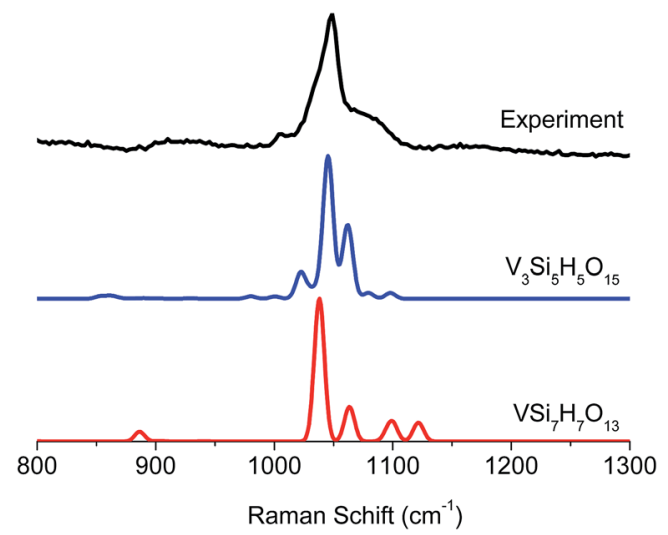

Fig. 9 Comparison between experimental (black) and calculated $\mathrm{VSi}_{7} \mathrm{H}_{7} \mathrm{O}_{13}$ (red) and $\mathrm{V}_{3} \mathrm{Si}_{5} \mathrm{H}_{5} \mathrm{O}_{15}$ (blue) rRaman spectra.

Si-O-V structural motifs (cyan). On the other hand, the signals at 1050 and 1075 $\mathrm{cm}^{-1}$ are primarily dominated by states involving the $\mathrm{V}=\mathrm{O}$ and $\mathrm{Si}-\mathrm{O}-\mathrm{V}$ fragments (cyan and purple). Furthermore, as is seen in Fig. 9 the experimental features at $1025 \mathrm{~cm}^{-1}$ are better reproduced by the trimeric $\mathrm{V}_{3} \mathrm{Si}_{5} \mathrm{H}_{5} \mathrm{O}_{15}$, rather than the monomeric $\mathrm{VSi}_{7} \mathrm{H}_{7} \mathrm{O}_{13}$ due to the dominating $\mathrm{V}-\mathrm{O}-\mathrm{V}$ vibrations.

\section{X-ray spectroscopy}

In an effort to obtain further insight into the predominant species, the V L-edge and $\mathrm{O}$ K-edge spectra of all models for both the molecular and the heterogeneous catalytic systems were calculated on the DFT/ROCIS level. It has been discussed recently ${ }^{24}$ that the shape of the $\mathrm{O}$ K-edge spectrum of $\mathrm{K}\left[\mathrm{VO}\left(\mathrm{O}_{2}\right)\right.$ Hheida], can be explained by considering the TD-DFT calculation molecular admixtures of $\left[\mathrm{VO}\left(\mathrm{O}_{2}\right) \text { Hheida }\right]^{-}$and the oxygen deficient $[\mathrm{VO}(\mathrm{O}) \text { Hheida }]^{-}$ions according to the relation: ${ }^{24}$

$$
\alpha^{*}\left[\mathrm{VO}\left(\mathrm{O}_{2}\right) \text { Hheida }\right]+(1-\alpha)^{*}[\mathrm{VO}(\mathrm{O}) \text { Hheida }]
$$

Moreover, a quantitative measure of the sample heterogeneity can be obtained when relation (1) is combined with the DFT-ROCIS calculated V L-edge and O Kedge spectra. The corresponding spectra for $\alpha$-values ranging between 1 and 0 are presented in Fig. 10.

Subsequently, these spectra were used to simultaneously fit the $\mathrm{V}_{3}$-edge and O K-edge spectra parts, according to a fitting procedure, described in the ESI. $\dagger$ The best agreement between theory and experiment is observed for $\alpha=0.8$ (highlighted red in Fig. 10), indicating a 20\% admixture of the oxygen deficient species. Additional information can be obtained by analyzing the relativistic many particle states in terms of contributions arising from the oxo, the peroxo and the ligand oxygen functional groups, as well as the most important single electron excitations. This has been proven to be useful in describing bonding features for both molecular complexes ${ }^{17}$ and solids. ${ }^{20}$ As can be seen in Fig. 4 (middle) all three groups strongly participate in the intensity mechanism of both V L-edge and O K-edge spectra, due to the $20 \%$ admixture of the [VO(O)Hheida $]^{-}$ 


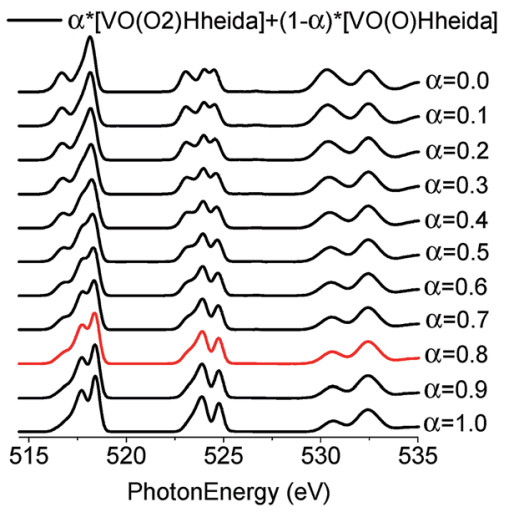

Fig. $10 \mathrm{~B} 3 \mathrm{LYP} / \mathrm{ROCIS}$ calculated spectra for admixtures of $\left[\mathrm{VO}\left(\mathrm{O}_{2}\right) \mathrm{Hheida}^{-}\right.$and the oxygen deficient [ $\mathrm{VO}(\mathrm{O}) \mathrm{Hheida}]^{-}$ions according to the relation (1) for $\alpha$ values ranging between 1 and 0 . The red spectrum at $\alpha=0.8$ indicates the best agreement between theory and experiment.

species, as well as the strongly anisotropic environment around the $\mathrm{VO}_{6}$ vanadium centers. In particular, oxo, peroxo and ligand oxygen atoms dominate the main experimental features of the $\mathrm{O}$ K-edge spectrum located at 529 and $534 \mathrm{eV}$ in accordance with a previous analysis. ${ }^{24}$ Moreover, both bands are dominated by combinations of $\sigma$ - and $\pi$-single electron excitations $2 \mathrm{p} \rightarrow 3 \mathrm{~d}_{z^{2}}, 2 \mathrm{p} \rightarrow 3 \mathrm{~d}_{x^{2}-y^{2} / x y}$, and $2 \mathrm{p} \rightarrow 3 \mathrm{~d}_{x z / y z}$. Furthermore, a similar analysis of the corresponding $\mathrm{V} \mathrm{L}_{3}$-edge reflects the $\pi$-character of the signals located at 515 and $518 \mathrm{eV}$, as well as the $\sigma$-character of the signal positioned at $519 \mathrm{eV}$ and the mixed $\sigma$-and $\pi$-character of the shoulder centered at $516 \mathrm{eV}$.

In following, the same protocol is applied to the more challenging case of silica supported vanadia catalysts. The resulting V L-edge spectra are shown in Fig. S6 and $\mathrm{S} 7 \dagger$ for $2 \%$ and $8 \% \mathrm{~V}$ loadings respectively. By inspection of Fig. S6 and S7, $\dagger$ it becomes evident that the A and B-domain types of structures containing $\mathrm{V}-\mathrm{O}-\mathrm{V}$ bridges $\left(\mathrm{V}_{2} \mathrm{Si}_{6} \mathrm{H}_{6} \mathrm{O}_{14}, \mathrm{~V}_{2} \mathrm{Si}_{12} \mathrm{H}_{12} \mathrm{O}_{23}, \mathrm{~V}_{3} \mathrm{Si}_{5} \mathrm{H}_{5} \mathrm{O}_{15}\right.$ and $\left.\mathrm{V}_{4} \mathrm{Si}_{4} \mathrm{H}_{4} \mathrm{O}_{16}\right)$, provide the best agreement for both vanadium loadings, in terms of relative intensities and intensity distribution, especially for the $\mathrm{L}_{3}$ resonances located at 516, 517.5 and $519 \mathrm{eV}$. In addition, the $\mathrm{V}_{3} \mathrm{Si}_{5} \mathrm{H}_{5} \mathrm{O}_{15}$ calculated spectrum reproduces equally well both the $2 \%$ and the $8 \%$ vanadium loading spectra, reflecting the limited oligomerization at these levels of vanadium loading. Evidently by averaging over the above four structures (Fig. $\mathrm{S} 8 \dagger$ ) the average spectrum has a closer resemblance with the $\mathrm{V}_{2} \mathrm{O}_{5}$ spectrum. On the other hand, the 'open' trimeric structure $\mathrm{V}_{3} \mathrm{Si}_{11} \mathrm{H}_{15} \mathrm{O}_{37}$ does not show relevant agreement, indicating the preference of $\mathrm{VO}_{x}$ to form more strain configurations. This is in accordance with the recent observation for silica supported molybdena catalysts that showing that such a strain effect has been directly associated with an increase in reactivity. ${ }^{38}$ Collectively, by combining the results from the three individual spectroscopic experiments: rRaman, V L-edge and O K-edge (Fig. 9, 11 and S9†) it is the trimeric structure $\mathrm{V}_{3} \mathrm{Si}_{5} \mathrm{H}_{5} \mathrm{O}_{15}$ that provides consistently the best possible agreement. Furthermore, it should be emphasized that the $\mathrm{V}_{3} \mathrm{Si}_{5} \mathrm{H}_{5} \mathrm{O}_{15}$ structure provides in addition very good agreement for the relative intensities of the $\mathrm{V} \mathrm{L}$-edge and $\mathrm{O}$ K-edge spectra 


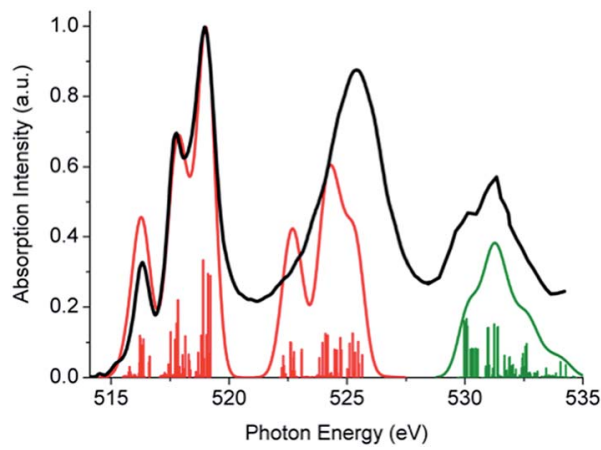

Fig. 11 DFT/ROCIS (B3LYP/def2-TZVP), V L-edge (red solid line) and O K-edge (green solid line) calculated spectra for $\mathrm{V}_{3} \mathrm{Si}_{5} \mathrm{H}_{5} \mathrm{O}_{15}$. The black thick line represents the background-subtracted experimental powder spectrum. Red and green stick lines correspond to SOC-corrected states. All calculated spectra were energy shifted. A constant line shape broadening of $0.8 \mathrm{eV}$ was applied.

(Fig. 11). This is important as in contrast to the situation with $\mathrm{V}_{2} \mathrm{O}_{5}$, these two edge spectra do not provide complementary information about the $\mathrm{V}-\mathrm{O}$ bonds since there are additional $\mathrm{Si}-\mathrm{O}$ bonds that lead to recognizable features in the spectrum. Hence, it appears that this trimeric structure provides the best balance between vanadyl bonds $\mathrm{V}=\mathrm{O}$ and bridging units $\mathrm{V}-\mathrm{O}-\mathrm{V}, \mathrm{Si}-\mathrm{O}-\mathrm{V}$ and $\mathrm{Si}-\mathrm{O}-\mathrm{Si}$ which is of paramount importance for correct spectral predictions.

\section{Degree of oligimerization}

As a final step, the effect of the oligomerization on the calculated spectra was investigated. Following the same protocol as for the molecular catalyst, the analysis was accomplished by a simultaneous fit of the weighted superposition of all calculated spectra to the $\mathrm{V} \mathrm{L}_{3}$-edge experimental spectrum (V loading of $2 \%$ ). It should be highlighted that the $\mathrm{V} \mathrm{L}_{3}$-spectrum serves as a good candidate for this analysis as it is composed of three clearly visible signals. By contrast, the respective Raman and $\mathrm{O}$ K-edge spectra were not used in the fitting due to the relatively broad nature of the spectral features. These spectra were used however, in the second step of the analysis in order to validate the fitting results. Initially, the fit was performed over the whole dataset. It was found however that better fits

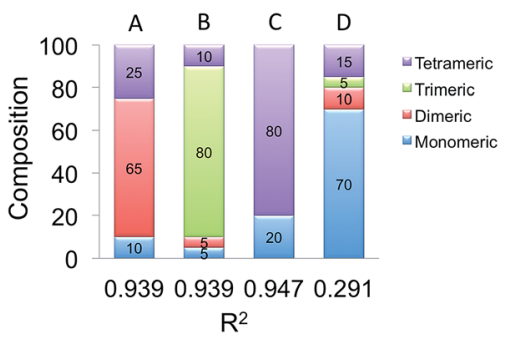

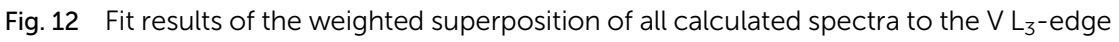
experimental spectrum. Cases (A), (B), (C) represent local minima of the fit function corresponding to the best $R^{2}$ values achievable by starting the fit from a pure dimer (A), trimer $(B)$, tetramer $(C)$ or monomer (D) structure. 


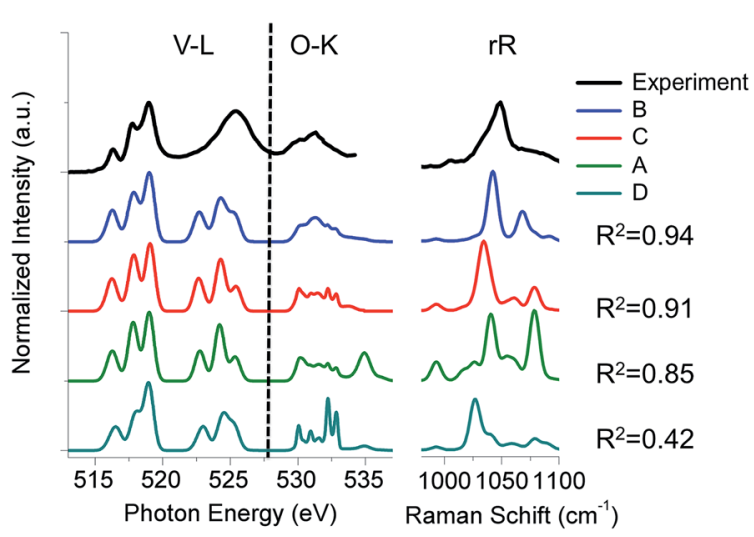

Fig. 13 Experimental (black) versus calculated weighted $\mathrm{V}$ L-edge, $\mathrm{O} \mathrm{K}$-edge and resonance Raman spectra. Weighting schemes, (A): 10\% monomeric, $65 \%$ dimeric and $25 \%$ tetrameric (green), (B): 5\% monomeric, 5\% dimeric, $80 \%$ trimeric and $10 \%$ tetrameric (blue), (C): $20 \%$ monomeric and $80 \%$ tetrameric (red) and (D): $70 \%$ monomeric, $10 \%$ dimeric, $5 \%$ trimeric and $15 \%$ tetrameric (cyan). The corresponding $R^{2}$ values are provided at the righthand side.

could be obtained by considering only the monomeric $\left(\mathrm{VSi}_{7} \mathrm{H}_{7} \mathrm{O}_{13}, \mathrm{VSi}_{7} \mathrm{H}_{9} \mathrm{O}_{13}\right.$ and $\left.\mathrm{VSi}_{7} \mathrm{H}_{11} \mathrm{O}_{13}\right)$, dimeric $\left(\mathrm{V}_{2} \mathrm{Si}_{6} \mathrm{H}_{6} \mathrm{O}_{14}\right.$ and $\left.\mathrm{V}_{2} \mathrm{Si}_{12} \mathrm{H}_{12} \mathrm{O}_{23}\right)$, trimeric $\left(\mathrm{V}_{3} \mathrm{Si}_{5} \mathrm{H}_{5} \mathrm{O}_{15}\right)$ and tetrameric $\left(\mathrm{V}_{4} \mathrm{Si}_{4} \mathrm{H}_{4} \mathrm{O}_{16}\right)$ model structures. The results of the fitting are visualized in Fig. 12, while the corresponding weighted $\mathrm{O}$ K-edge and resonance Raman spectra together with the respective V L-edge spectra are shown in Fig. 13.

The B weighting scheme involving $5 \%$ monomeric, $5 \%$ dimeric, $80 \%$ trimeric and $10 \%$ tetrameric structures provides the best agreement with all the three spectra, V L-edge, O K-edge and rRaman $\left(R^{2}=0.94\right)$. The respective weighting schemes D, A and C provide significantly worse agreement $\left(R^{2}=0.42,0.85\right.$ and 0.91 , respectively). Thus, the combined analysis of three different spectroscopic methods demonstrates that under limited vanadium loading conditions, trimeric structures are dominant. This is in agreement with recent computational studies that address the reactivity of these systems. ${ }^{39}$

\section{Conclusions}

In conclusion, a systematic computational protocol based on advanced theoretical spectroscopy was applied in order to extract the structural information of active catalytic centers in both homogenous and heterogeneous catalysis. Establishing detailed structure-spectra relationships requires the simultaneous interpretation of the complete spectroscopic response of the catalytic material. To the best of our knowledge, this work represents the first study of this kind. The approach was validated to perform equally well with both homogeneous and heterogeneous catalytic cases. In particular the simultaneous analysis of the available experimental absorption, rRaman as well as V L-edge and O K-edge XAS spectra provided detailed insights into the geometric and electronic structural origin of the observed spectral features. In the case of the $\mathrm{K}\left[\mathrm{VO}\left(\mathrm{O}_{2}\right)\right.$ Hheida $]$ catalyst, the combined analysis revealed that the low-lying absorption LMCT 
band, as well as the rRaman profile of this excited chromophore, are dominated by the actual $\left[\mathrm{VO}\left(\mathrm{O}_{2}\right) \text { Hheida }\right]^{-}$complex. On the other hand the respective XAS spectra contain a $20 \%$ admixture of the oxygen deficient $[\mathrm{VO}(\mathrm{O}) \text { Hheida }]^{-}$species. The same protocol was applied to interpret the spectroscopic response of the heterogeneous silica-supported vanadium oxide $\mathrm{VO}_{x} / \mathrm{SBA}-15$ catalyst. It was shown that under limited vanadium loadings molecular species with predominant trimeric structures provide the best agreement between theory and experiment for all the available spectra. Although a identification of a catalytic center in a heterogeneous catalyst is a much more complicated task, having a tool with predictive power forms an excellent basis for the design of dedicated spectroscopic experiments. Further experimental and theoretical studies based on multiwavelength rRaman and X-ray measurements, as well as high-level theory, are currently underway in order to determine the reaction mechanism of these catalysts.

\section{Acknowledgements}

The authors gratefully acknowledge financial support from the Max Planck Society. The Helmholtz-Zentrum Berlin (HZB) staff are acknowledged for continuously supporting the synchrotron based near ambient pressure spectroscopy experiments of the Fritz Haber Institute at BESSY II. We thank Dr Philipp Gruene, Dr Till Wolfram, and Daniel Brennecke for preparation of the supported vanadium catalysts and Dr Olaf Timpe, Dr Johannes Noack, and Anne Kärgel for preparation of the molecular complex. Raman spectroscopy was performed by $\mathrm{Dr}$ Philipp Gruene, Dr Oliver Korup, and Dr Johannes Noack. UV-vis spectra were taken by Dr Genka Tzolova-Müller. X-ray absorption spectra were recorded by Dr Michael Hävecker and Dr Till Wolfram. The reviewers of the manuscript are gratefully acknowledged for their constructive comments.

\section{Notes and references}

1 R. Schloegl, Nat. Mater., 2008, 7, 772-774.

2 T. W. Hansen, J. B. Wagner, P. L. Hansen, S. Dahl, H. Topsøe and C. J. H. Jacobsen, Science, 2001, 294, 1508-1510.

3 J. K. Norskov, T. Bligaard, J. Rossmeisl and C. H. Christensen, Nat. Chem., 2009, 1, 37-46.

4 T. Bligaard, J. K. Nørskov, S. Dahl, J. Matthiesen, C. H. Christensen and J. Sehested, J. Catal., 2004, 224, 206-217.

5 A. A. Balandin, in Advances in Catalysis, ed. H. P. D. D. Eley and B. W. Paul, Academic Press, 1969, vol. 19, pp. 1-210.

6 T. Petrenko, S. DeBeer George, N. Aliaga-Alcalde, E. Bill, B. Mienert, Y. Xiao, Y. Guo, W. Sturhahn, S. P. Cramer, K. Wieghardt and F. Neese, J. Am. Chem. Soc., 2007, 129, 11053-11060.

7 J. F. Berry, E. Bill, E. Bothe, S. D. George, B. Mienert, F. Neese and K. Wieghardt, Science, 2006, 312, 1937-1941.

8 J. C. Schöneboom, F. Neese and W. Thiel, J. Am. Chem. Soc., 2005, 127, 58405853.

9 S. Ye, C.-Y. Geng, S. Shaik and F. Neese, Phys. Chem. Chem. Phys., 2013, 15, 8017-8030. 
10 S. Ye and F. Neese, Proc. Natl. Acad. Sci. U. S. A., 2011, 108, 1228-1233.

11 K. M. Lancaster, M. Roemelt, P. Ettenhuber, Y. Hu, M. W. Ribbe, F. Neese, U. Bergmann and S. DeBeer, Science, 2011, 334, 974-977.

12 D. A. Pantazis, W. Ames, N. Cox, W. Lubitz and F. Neese, Angew. Chem., Int. Ed., 2012, 51, 9935-9940.

13 N. Cox, M. Retegan, F. Neese, D. A. Pantazis, A. Boussac and W. Lubitz, Science, 2014, 345, 804-808.

14 J. E. Molinari and I. E. Wachs, J. Am. Chem. Soc., 2010, 132, 12559-12561.

15 C. Hess, ChemPhysChem, 2009, 10, 319-326.

16 D. Maganas, M. Roemelt, M. Havecker, A. Trunschke, A. Knop-Gericke, R. Schlogl and F. Neese, PCCP Phys. Chem. Chem. Phys., 2013, 15, 7260-7276.

17 D. Maganas, M. Roemelt, T. Weyhermuller, R. Blume, M. Havecker, A. KnopGericke, S. DeBeer, R. Schlogl and F. Neese, PCCP Phys. Chem. Chem. Phys., 2014, 16, 264-276.

18 M. Roemelt, D. Maganas, S. DeBeer and F. Neese, J. Chem. Phys., 2013, 138, 204101.

19 M. Roemelt and F. Neese, J. Phys. Chem. A, 2013, 117, 3069-3083.

20 D. Maganas, S. DeBeer and F. Neese, Inorg. Chem., 2014, 53, 6374-6385.

21 T. Petrenko and F. Neese, J. Chem. Phys., 2012, 137, 234107.

22 T. Petrenko and F. Neese, J. Chem. Phys., 2007, 127, 164112.

23 P. Gruene, T. Wolfram, K. Pelzer, R. Schlogl and A. Trunschke, Catal. Today, 2010, 157, 137-142.

24 L. L. Sun, K. E. Hermann, J. Noack, O. Timpe, D. Teschner, M. Hävecker, A. Trunschke and R. Schlögl, J. Phys. Chem. C, 2014, 118, 24611-24622.

25 A. E. Stiegman, J. Phys. Chem. C, 2011, 115, 10917-10924.

26 Z. Wu, S. Dai and S. H. Overbury, J. Phys. Chem. C, 2009, 114, 412-422.

27 D. E. Keller, T. Visser, F. Soulimani, D. C. Koningsberger and B. M. Weckhuysen, Vib. Spectrosc., 2007, 43, 140-151.

28 N. Magg, B. Immaraporn, J. B. Giorgi, T. Schroeder, M. Bäumer, J. Döbler, Z. Wu, E. Kondratenko, M. Cherian, M. Baerns, P. C. Stair, J. Sauer and H.-J. Freund, J. Catal., 2004, 226, 88-100.

29 C. Moisii, M. D. Curran, L. J. van de Burgt and A. E. Stiegman, J. Mater. Chem., 2005, 15, 3519-3524.

30 C. Moisii, L. J. van de Burgt and A. E. Stiegman, Chem. Mater., 2008, 20, 39273935.

31 J. P. Thielemann, T. Ressler, A. Walter, G. Tzolova-Mueller and C. Hess, Appl. Catal., A, 2011, 399, 28-34.

32 J. N. J. van Lingen, O. L. J. Gijzeman, B. M. Weckhuysen and J. H. van Lenthe, J. Catal., 2006, 239, 34-41.

33 J. Döbler, M. Pritzsche and J. Sauer, J. Phys. Chem. C, 2009, 113, 12454-12464.

34 D. Maganas, M. Roemelt, M. Havecker, A. Trunschke, A. Knop-Gericke, R. Schlogl and F. Neese, Phys. Chem. Chem. Phys., 2013, 15, 7260-7276.

35 D. Maganas, M. Roemelt, T. Weyhermuller, R. Blume, M. Havecker, A. KnopGericke, S. DeBeer, R. Schlogl and F. Neese, Phys. Chem. Chem. Phys., 2014, 16, 264-276.

36 M. Cavalleri, K. Hermann, A. Knop-Gericke, M. Haevecker, R. Herbert, C. Hess, A. Oestereich, J. Doebler and R. Schloegl, J. Catal., 2009, 262, 215-223. 
37 M. Baron, H. Abbott, O. Bondarchuk, D. Stacchiola, A. Uhl, S. Shaikhutdinov, H.-J. Freund, C. Popa, M. V. Ganduglia-Pirovano and J. Sauer, Angew. Chem., Int. Ed., 2009, 48, 8006-8009.

38 K. Amakawa, L. Sun, C. Guo, M. Haevecker, P. Kube, I. E. Wachs, S. Lwin, A. I. Frenkel, A. Patlolla, K. Hermann, R. Schloegl and A. Trunschke, Angew. Chem., Int. Ed., 2013, 52, 13553-13557.

39 X. Rozanska, R. Fortrie and J. Sauer, J. Am. Chem. Soc., 2014, 136, 7751-7761. 